\title{
The Role of Hierarchies in Exploratory Data Mining
}

\author{
Raghu Ramakrishnan \\ Yahoo! Research, Santa Clara, CA, USA
}

In a broad range of data mining tasks, the fundamental challenge is to efficiently explore a very large space of alternatives. The difficulty is two-fold: first, the size of the space raises computational challenges, and second, it can introduce data sparsity issues even in the presence of very large datasets. In this talk, well consider how the use of hierarchies (e.g., taxonomies, or the OLAP multidimensional model) can help mitigate the problem. 